\title{
Differential association of microRNAs with polysomes reflects distinct strengths of interactions with their mRNA targets
}

\author{
NATALI MOLOTSKI and YOAV SOEN ${ }^{1}$ \\ Department of Biological Chemistry, Weizmann Institute of Science, Rehovot 76100, Israel
}

\begin{abstract}
While microRNAs have been shown to copurify with polysomes, their relative fraction in the translation pool (polysome occupancy) has not yet been measured. Here, we introduce a high-throughput method for quantifying polysome occupancies of hundreds of microRNAs and use it to investigate factors affecting these occupancies. Analysis in human embryonic stem cells (hESCs) and foreskin fibroblasts (hFFs) revealed microRNA-specific preferences for low, medium, or high polysome occupancy. Bioinformatics and functional analysis based on overexpression of endogenous and chimeric microRNAs showed that the polysome occupancy of microRNAs is specified by its mature sequence and depends on the choice of seed. Nuclease treatment further suggested that the differential occupancy of the microRNAs reflects interactions with their mRNA targets. Indeed, analysis of microNRA॰mRNA duplexes showed that pairs involving high occupancy microRNAs exhibit significantly higher binding energy compared to pairs with low occupancy microRNAs. Since mRNAs reside primarily in polysomes, strong interactions lead to high association of microRNAs with polysomes and vice versa for weak interactions. Comparison between hESCs and hFFs data revealed that hESCs tend to express lower occupancy microRNAs, suggesting that cell type-dependent translational features may be affected by expression of a particular set of microRNAs.
\end{abstract}

Keywords: microRNA; polysomes occupancy of microRNAs; high-throughput analysis; human embryonic stem cells; free energy

\section{INTRODUCTION}

MicroRNAs have been shown to regulate a wide range of biological processes by destabilizing messenger RNAs and by repressing the translation of these mRNAs (Ambros 2004; Bartel and Chen 2004; Bagga et al. 2005; Lim et al. 2005; Carthew 2006; Jackson and Standart 2007; Meister 2007; Nilsen 2007; Filipowicz et al. 2008; Guo et al. 2010). Target specificity and efficacy of repression by microRNAs are influenced by multiple features of the microRNA and its targets (Grimson et al. 2007). It has been shown that the main determinants of targeting are based on recognition between bases $2-8$ at the $5^{\prime}$ end of the microRNA and a matching sequence (seed) on the $3^{\prime}$ UTR of its targets. These determinants were shown to specify mRNA targets for degradation (Doench and Sharp 2004; Brennecke et al. 2005; Lim et al. 2005; Grimson

\footnotetext{
${ }^{1}$ Corresponding author

E-mail yoavs@weizmann.ac.il

Article published online ahead of print. Article and publication date are at http://www.rnajournal.org/cgi/doi/10.1261/rna.033142.112.
}

et al. 2007; Nielsen et al. 2007; Guo et al. 2010) and for translation repression (Baek et al. 2008; Selbach et al. 2008).

Involvement of microRNAs in repression of translation suggests that they might be associated with polysomes. Indeed, previous work based on velocity sedimentation in sucrose gradient reported copurification of several microRNAs with polysomes (Olsen and Ambros 1999; Kim et al. 2004; Nelson et al. 2004; Maroney et al. 2006; Nottrott et al. 2006; Jannot et al. 2011). It has also been hypothesized that association of microRNAs with polysomes reflects involvement of these microRNAs with repression of translation (Olsen and Ambros 1999; Nelson et al. 2004; Nottrott et al. 2006; Petersen et al. 2006; Wang et al. 2008). It is, in fact, plausible that the ability of any microRNA to repress translation depends on its degree of association with polysomes (i.e., its relative fraction in the translational pool). However, the amount of microRNA in polysomes relative to its total amount has not yet been quantified. Consequently, the relative tendency of specific microRNAs to associate with the translational pool remains unknown and so are the factors that influence this association. 
Here, we measure polysomal association of over a hundred microRNAs by coupling velocity sedimentation fractionation with high-throughput measurements of microRNA levels. These measurements identified microRNA-specific preferences for low, medium, or high association with polysomes, denoted as "microRNA polysome occupancy." We show that this preference is specified by the mature form of the microRNA, influenced by its seed sequence, and is likely caused by microRNA-specific differences in the energy of pairing between the microRNA and its mRNA targets. normalization, we computed the occupancy of each microRNA by dividing its normalized levels in the polysomal pool by the sum of its normalized levels in both pools (Fig. 1A).

Subsequent analysis was restricted to microRNAs detected, for each cell type, at qPCR cycles below $35\left(\mathrm{C}_{\mathrm{T}}<35\right)$ in both polysome fractions (ribosome-free and polysome-bound). Of the 667 measured microRNAs, 123 and 158 microRNAs passed these criteria in hESCs and hFFs, respectively, and 77 microRNAs passed the criteria in both cell types.

Comparison of microRNA occupancy measurements in replicate samples of hESCs revealed high reproducibility, slightly below that of standard microRNA expression measurements from the same samples $\left(\mathrm{R}^{2}=0.84\right.$, correlation $=$

\section{RESULTS}

\section{Establishing a high-throughput method of measuring polysome occupancy of microRNAs}

We developed a protocol for analyzing polysome occupancy of microRNAs using density gradient sedimentation followed by high-throughput analysis of small RNAs in different fractions of the gradient (Fig. 1A). This protocol extends the method of profiling mRNA association with polysomes (Arava et al. 2003; Hendrickson et al. 2009; Melamed et al. 2009) so as to make it applicable to microRNAs.

We tested the method in human embryonic stem cells (hESCs) (line H9) and foreskin fibroblasts (hFFs). Following extract fractionation by density gradient centrifugation, we collected the ribosomefree (unbound) fraction into one pool, and the polysomal fractions into another pool (the $80 \mathrm{~S}$ fraction was excluded to enable better separation between the pools). We then isolated total RNA from each pool and measured the levels of 667 microRNAs by real-time qPCR using microfluidic arrays of TaqMan microRNA probes (Chen et al. 2005). To normalize the measurements of each microRNA, we spiked into each pool (prior to RNA extraction) $1.3 \mathrm{ng} / \mu \mathrm{L}$ of exogenous control microRNA. For the exogenous control, we selected miR- $488^{*}$, whose endogenous expression was below the detectable level as determined by realtime qPCR using a specific Taqman assay. Normalization was performed by dividing the measured levels of each microRNA in each pool by the levels of (the spiked-in) miR-488*. Following

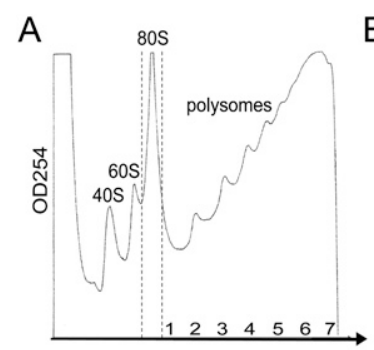

Sedimentation along sucrose gradient

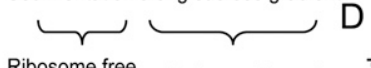

B
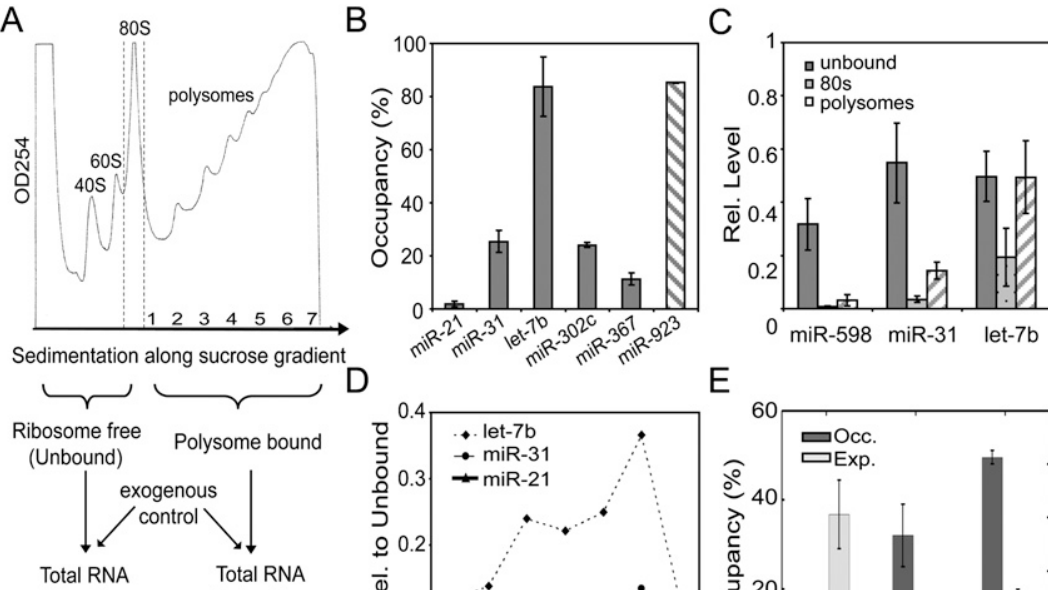

$\begin{array}{ll}\text { Total RNA } & \text { Total RNA } \\ \text { Extraction } & \text { Extraction }\end{array}$
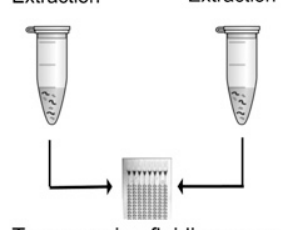

Taqman microfluidic arrays

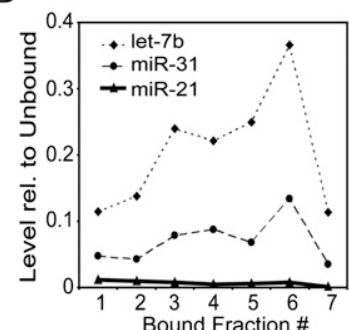

$\mathrm{E}$

$\mathrm{F}$
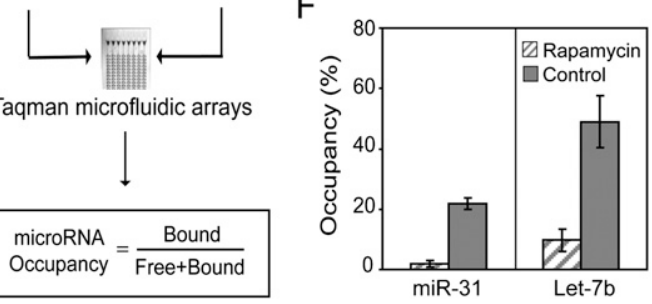

Occupancy $=\overline{\text { Free+Bound }}$

FIGURE 1. High-throughput method for measuring polysome occupancy of microRNAs. $(A)$ Schematics of the approach. MicroRNA occupancies were computed based on the levels measured in the "polysome-bound" and "ribosome-free" fractions as indicated at the bottom. (B) Occupancy values for a few microRNAs, including miR-923 (right bar), which was shown to be a fragment of the $28 \mathrm{~S}$ rRNA (Griffiths-Jones 2006, 2007; Griffiths-Jones et al. 2008). (C) Unbound, 80S, and polysomal fractions of high (let-7b), medium (miR-31), and low (miR598) occupancy microRNAs indicates primary association with either the unbound or polysome fractions. $(D, E)$ Validation of the high-throughput measurement by higher resolution profiles of representative microRNAs in hFFs $(D)$ and using individual, microRNA-specific assays $(E)$. Shown in $D$ are levels of three microRNAs in seven equal volume (1-mL) fractions within the polysome-bound pool. Fractions are indicated along the OD profile in A. Displayed levels are relative to the levels detected in the ribosome-free, unbound pool (Unbound). Shown in $E$ are occupancy (left $y$-axis, dark gray) and relative expression (right $y$-axis, light gray) for miR-21, miR-31, and let-7b, each measured using individual, microRNA-specific Taqman assays. (F) Occupancy of two representative microRNAs, miR-31 and let-7b, following treatment with $100 \mathrm{nM}$ of rapamycin or DMSO (control). Occupancies were computed based on the polysomebound and ribosome-free fractions as illustrated in $A$. Results in $B, C, E$, and $F$ are displayed as mean $+/-$ standard error (SE). 
0.91 compared, respectively, with $\mathrm{R}^{2}=0.91$ and correlation $=0.95)$ (Supplemental Fig. S1A,B). Interestingly, the occupancy of miR-923, a reported fragment of the $28 \mathrm{~S}$ rRNA (Griffiths-Jones 2006, 2007; Griffiths-Jones et al. 2008), was above $80 \%$ (Fig. 1B).

To verify that the exclusion of the $80 \mathrm{~S}$ fraction did not lead to a significant change in the assessment of polysome occupancy, we measured the levels of a few microRNAs in this fraction. The levels of these microRNAs in the $80 \mathrm{~S}$ fraction were significantly lower compared with their levels in both the unbound and polysome fractions (Fig. 1C), indicating that the results should not be strongly affected by the exclusion of the $80 \mathrm{~S}$ fraction.

The fidelity of our occupancy estimation was further evaluated in human foreskin fibroblasts by measuring the levels of three representative microRNAs, miR-21, miR-31, and let-7b, in eight individual fractions of the sucrose gradient (one unbound and seven bound fractions). The resulting high-resolution polysome profiles of these microRNAs (Fig. 1D) were consistent with the low, medium, and high occupancy levels measured, respectively, for these microRNAs by the pooling method of Figure 1A (Fig. 1B). Measurements of microRNA occupancy and expression using individual qPCR probes confirmed the differences in occupancy measured using the microfluidic Taqman arrays. It also showed that highly expressed microRNAs, such as miR21 and let-7b, can exhibit very different polysome occupancies (Fig. 1E).

To verify that high occupancy levels of microRNAs reflect sedimentation with ribosomes and not with other protein complexes, we measured the occupancy of representative microRNAs following treatment with rapamycin which leads to polysome dissociation (Supplemental Fig. S2; Terada et al. 1994; Raught et al. 2001). A 4-h treatment of hFFs with $100 \mathrm{nM}$ of rapamycin, indeed, led to significant reduction in the polysome occupancy of miR-31 and let- $7 \mathrm{~b}$ (Fig. 1F), consistent with association of microRNAs with polysomes.

\section{microRNAs exhibit distinct degrees of association with polysomes}

Following the establishment of the high-throughput assay, we used it to investigate factors affecting the occupancy of microRNAs in hESCs and hFFs, representing undifferentiated and fully differentiated cells, respectively. For each of these samples, we analyzed the polysome occupancy and expression level of the microRNAs using the same highthroughput Taqman assay.

Figure 2A displays distributions of microRNA occupancies measured for hESCs and hFFs. It reveals a very broad range of occupancies with the most frequent value at $\sim 20 \%$. Side-by-side examination of occupancies of 77 microRNAs that were commonly detected in hESCs and hFFs revealed very similar occupancies for most microRNAs (Fig. 2B;
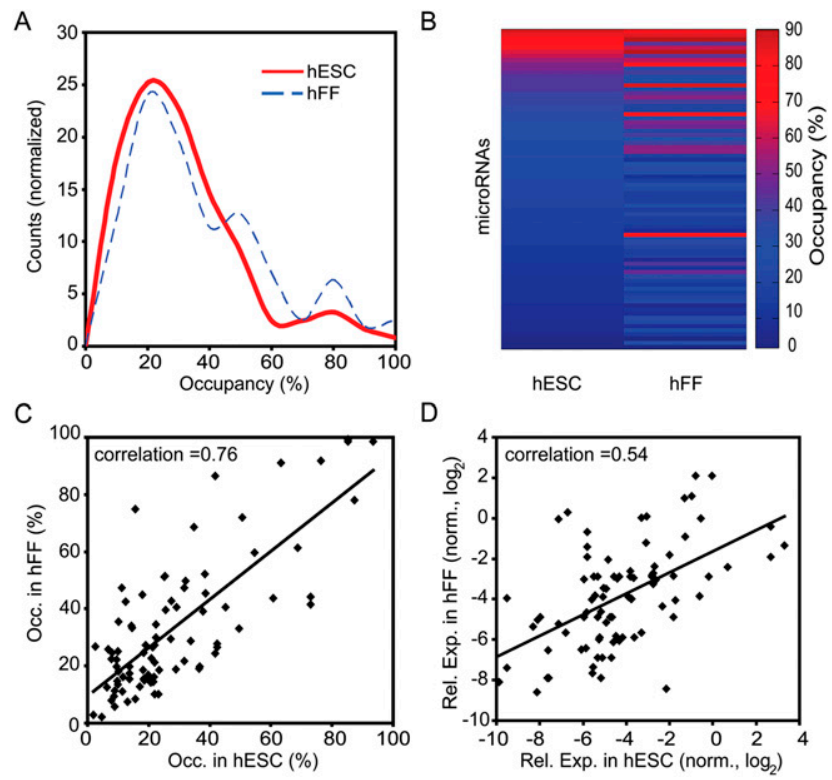

FIGURE 2. Dependence of microRNA occupancy on cellular context. (A) Distributions of microRNA occupancies evaluated in undifferentiated human embryonic stem cells (hESCs) and human foreskin fibroblasts (hFFs). (B) Color-coded heat map displaying side-by-side measurements of microRNA occupancies in hESCs and hFFs. $(C, D)$ Correlation between hESC and hFF microRNAs with respect to occupancy $(C)$ and abundance $(D)$. Note the higher correlation with respect to occupancy ( $C$ vs. $D, P$-value $=0.009$ based on Fisher's r-to- $z$ transformation).

microRNAs exhibiting differences in occupancy are listed in Supplemental Appendix). In addition, the correlation between microRNA occupancies in hESCs and hFFs was significantly higher than the respective correlation between the microRNA expression levels in these cell types ( 0.76 vs. $0.54 ; P$-value $=0.009$ based on Fisher's r-to-z transformation) (Fig. 2C,D). This suggests that polysome occupancy of microRNAs is less sensitive to changes in the cellular context (compared to the sensitivity of microRNA expression).

\section{hESC-specific microRNAs exhibit a tendency toward low polysome occupancies}

To examine relations between microRNA occupancy and expression, we ranked the microRNAs that were detected in each sample by their expression levels and plotted the occupancies in that order (Fig. 3, left panels). This ordering showed that highly expressed microRNAs in undifferentiated hESCs tend to have lower occupancy levels compared with less abundant microRNAs (Fig. 3A). Quantitative analysis of microRNA occupancy and expression in hESCs revealed a clear inverse correlation $(-0.51)$ between these parameters (Fig. 3A, right panel). A slightly weaker (though statistically indistinguishable) correlation was observed in hESCs that have been induced to differentiate for $10 \mathrm{~d}$ with $0.5 \mu \mathrm{M}$ of retinoic acid (correlation $=-0.46$ ) (Supplemental Fig. S3). In the differentiated hFF cells, however, the 
A

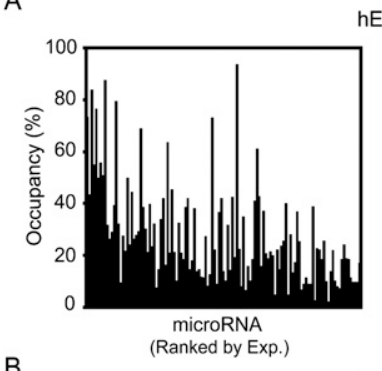

hESC
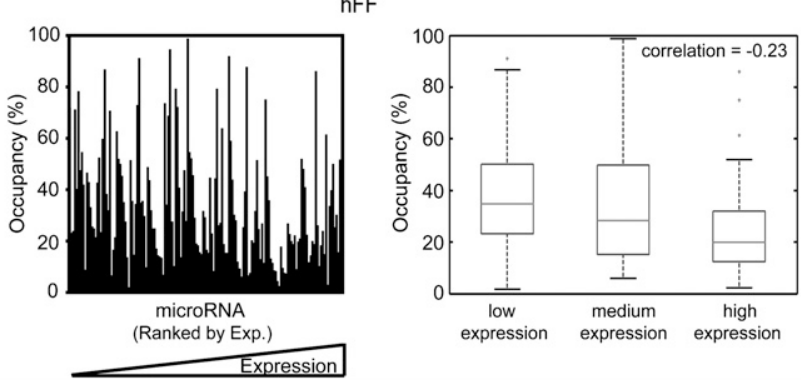

FIGURE 3. MicroRNAs that are highly expressed in hESCs have a stronger bias toward low occupancy compared to hFF microRNAs. (A) Left: Occupancy values for hESC microRNAs that are ranked according to their expression level. Right: Box plot distributions of occupancies of hESC microRNAs divided into groups of high, medium, and low expression. (B) Same as $A$ for hFFs. Note the significantly larger inverse correlation between occupancy and expression in hESCs compared to hFFs $(-0.51$ vs. $-0.23, P$-value $=0.0035$ based on Fisher's r-to-z transformation).

inverse correlation between abundance and occupancy of microRNAs was much weaker (correlation $=-0.23$ in hFFs vs. -0.51 in hESCs, $P$-value $=0.0035$ ) (Fig. $3 \mathrm{~B}$ ). These results suggest that hESCs express a set of microRNAs that is biased toward low polysome occupancies compared with the set of microRNA in differentiating cells.

\section{The mature form of the microRNA, but not its abundance, suffices to specify its preference for high or low occupancy}

To test if the mature form of the microRNA suffices to specify its preference for high and low occupancy, we overexpressed the mature form of representative microRNAs (let-7 and miR-598, respectively) in hESCs. Transfection of mature let-7b and miR-598 constructs resulted in $\sim 400$ fold increase in the measured levels of these microRNAs (Fig. 4A,B; absolute levels shown in Supplemental Fig. S4). Despite the strong overexpression, the final levels of these microRNAs did not exceed the endogenous levels of other high-abundance microRNAs, such as those of the endogenous let-7b in hFFs (Fig. 4A) or miR-302c in hESCs (Supplemental Fig. S4). Since let-7b and miR-598 are normally expressed at very low levels in hESCs, the pools of mature let-7b and miR-598 were dominated by the transfected constructs. Notably, the measured occupancies of these constructs were indistinguishable from the occupancies of their endogenous counterparts (Fig. 4A,B), suggesting that the occupancy is largely independent of differences in the abundance of the mature microRNA (at least within the physiological range of abundance). Similarly, the occupancy of the endogenous let-7b in hFFs was comparable to the occupancy of this microRNA in hESCs despite the large (a few hundredfold) difference in the endogenous expression of let-7b in these cells. Altogether, these results indicate that the preference for high and low association of microRNAs with polysomes is strongly affected by features of the mature microRNA.

\section{Polysome occupancy of microRNAs is affected by the choice of seed}

To determine which part of the mature microRNA affects the preference toward low, medium, or high polysome occupancy, we analyzed the relative contributions of the microRNAs' seed and the remaining sequence (denoted here as the microRNA body). As a first step, we analyzed the difference in occupancy between hESC microRNAs harboring the same seed versus hESC microRNAs with nonidentical seeds. The average occupancy difference between microRNAs sharing the same seed was considerably lower than the average for microRNAs with different seeds $(5.8 \%$ vs.
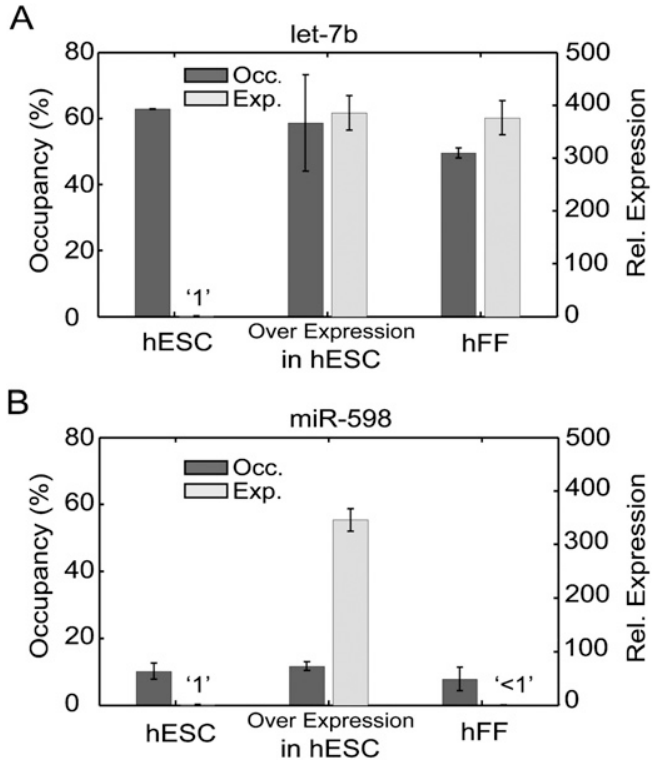

FIGURE 4. The mature form of the microRNA, but not its abundance, specifies its preference for low or high occupancy. Polysome occupancy and expression of let-7b $(A)$ and miR-598 (B) measured in (1) hESCs transfected with the mature form of the microRNAs (overexpression in hESC), (2) hESCs transfected with negative control pre-microRNA (hESC), and (3) in hFFs. The tendencies for high and low occupancy of let-7b and miR-598 in hESCs were unaffected by the overexpression and were similar to the occupancy in hFFs. Values displayed as mean $+/-$ SE. 
A

\begin{tabular}{|l|c|c|c|}
\cline { 2 - 3 } \multicolumn{1}{c|}{} & \multicolumn{2}{c|}{ Average difference } & \multicolumn{1}{c|}{} \\
\hline & Identical seeds & Non identical seeds & p value \\
\hline Occupancy $(\%)$ & 5.8 & 18.4 & $8.6 \mathrm{E}-14$ \\
\hline Expression $\left(\log _{2}\right)$ & 0.425 & 0.49 & 0.1 \\
\hline
\end{tabular}

B

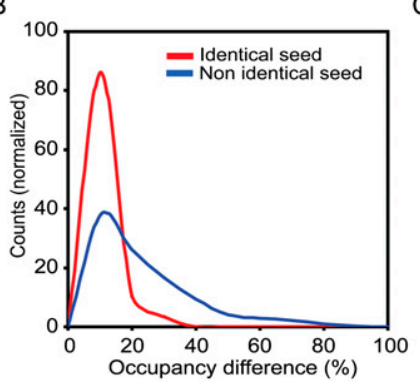

C

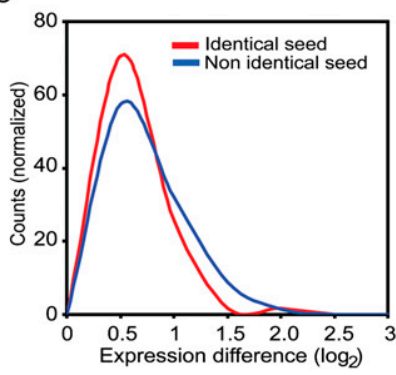

FIGURE 5. MicroRNAs harboring identical seeds exhibit similar polysome occupancies. (A) Comparison of polysome occupancy and expression values for microRNAs harboring the same seed vs. microRNAs with nonidentical seeds. $P$-values are based on the two-sample Kolmogorov-Smirnov test. (B) Normalized histograms of occupancy differences for pairs of microRNAs sharing the same seed (Identical seed), and pairs of microRNAs with different seeds (Nonidentical seed). (C) Same as $B$ for differences in expression as opposed to occupancy.

18.4\%, respectively; $P$-value $\left.=8.6 \times 10^{-14}\right)$ (Fig. $\left.5 \mathrm{~A}\right)$. Additionally, the distribution of occupancy differences within microRNAs sharing the same seed was significantly narrower compared with the distribution for nonidentical seeds (Fig. 5B). The distinction in occupancy difference within and outside seed groups was further emphasized by the lack of distinction with respect to microRNA expression $(P$-value $=0.1)($ Fig. 5A, bottom row; Fig. 5C; Supplemental Table S1). This analysis suggested a direct effect of the seed sequence on the association of microRNAs with polysomes. We verified this by functionally comparing the relative contributions of the microRNAs' seed and body. For that, we transfected hESCs with a high occupancy microRNA whose seed was replaced with the seed of a low occupancy microRNA, and vice versa for a low occupancy microRNA with a seed of a high occupancy microRNA (Fig. 6A). To simplify the analysis, we based it on two microRNAs, let-7b and miR-598, that are only weakly expressed in hESCs and exhibit, respectively, very high and very low polysome occupancy. Their chimeric versions were custom-made by Applied Biosystems, and their levels within each polysome fraction were measured using specially designed Taqman probes, also provided by Applied Biosystems. We verified that these probes can indeed detect the intended chimeric microRNAs (Supplemental Fig. S4) and distinguish them from the endogenous microRNAs (Supplemental Table S2). The measured occupancies of the chimeric microRNAs confirmed the hypothesized influence of the seed sequence (Fig. 6B). Indeed, the difference in occupancy between the high and low occupancy microRNAs, let-7b and miR598, was reversed by the seed swap; the polysome occupancy of let-7b harboring the seed of miR598 (chimera \#1) was $~ 3.5$ fold lower compared to the occupancy of miR598 with a seed of let-7b (chimera \#2). Taken together, bioinformatics and functional analyses show that the seed of the microRNA affects the occupancy more than the remaining part of the sequence.

\section{Differential polysome occupancy of microRNAs reflects differential strength of interactions with the mRNA targets}

The clear dependence of the microRNA occupancy on the seed sequence (Figs. 5, 6B) and the established role of the seed in target recognition suggested that the differential occupancy of microRNAs is caused by the interaction with their targets. To verify that, we treated cytoplasmic extracts from hESCs with micrococcal nuclease which digests exposed regions of the mRNA but not regions that are protected by ribosomes. As expected, nuclease digestion drastically decreased the polysome content, elevated the 80s fraction (Supplemental Fig. S5; Maroney et al. 2006) and led to a considerable decrease in the occupancy of let-7b, miR-31, and miR-21 representing, respectively, high, medium, and low occupancy microRNAs (Fig. 7A). Notably, despite the considerable increase in the $80 \mathrm{~S}$ fraction, all three microRNAs were detected almost exclusively in the unbound fraction and not in the $80 \mathrm{~S}$ fraction (Fig. 7B-D). This confirmed that the high polysome occupancy of microRNAs reflects their tendency to associate with exposed regions of the target mRNAs (e.g., the 3' UTR) and not with ribosomal RNA.
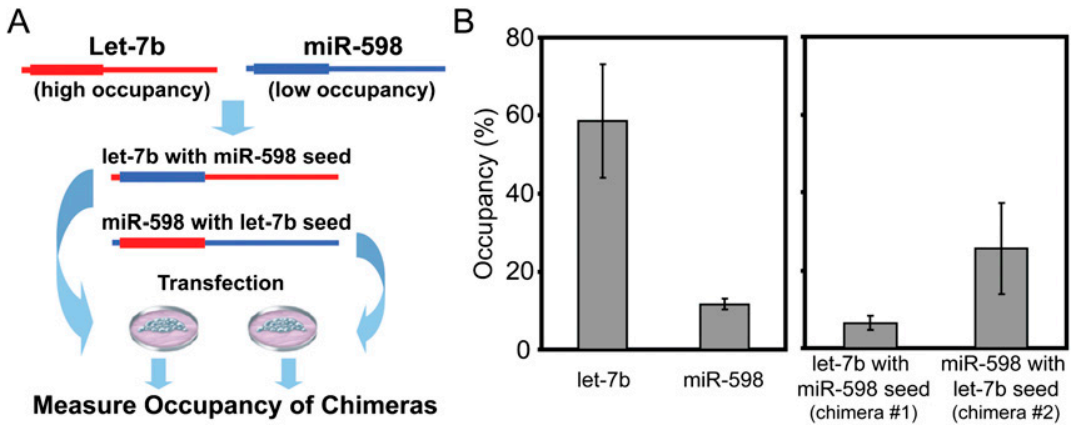

FIGURE 6. MicroRNA occupancy is functionally dependent on the choice of seed. $(A)$ Schematics of chimeric microRNAs designed for testing relative contributions of the microRNA seed and body to the degree of its association with polysomes. (B) Polysome occupancies of the endogenous (left) and chimeric microRNAs (right) that were transfected into hESCs. Note the reversal in the difference between the occupancies of let-7b and miR-598 upon exchange of seeds. Values displayed as mean $+/-$ SE. 
To investigate how the interaction with the mRNA targets can affect the polysome occupancy of microRNAs, we analyzed microRNA $\bullet$ RNA pairing interactions involving high and low occupancy microRNAs. We selected two groups of four microRNAs that exhibited very high $(>70 \%)$ or very low occupancy $(<13 \%)$ in both hESCs and hFFs. For these eight microRNAs, we analyzed the difference in free-energy scores $\left(\Delta \Delta \mathrm{G}, \Delta \mathrm{G}\right.$ _duplex, and $\Delta \mathrm{G}_{-}$open $)$computed by the PITA algorithm for each microRNA $\bullet$ mRNA pair (Kertesz et al. 2007; predicted targets for each microRNA are listed in Supplemental file, "Targets for ddG.xls"). This algorithm computes the gain in free energy $(\Delta \Delta G)$ due to binding of the microRNA, taking into account the need to open the structure of the mRNA ( $\Delta$ G_open) and the binding energy ( $\Delta G_{-}$duplex). Negative $\Delta \Delta G$ scores are associated with energetically favorable interactions. Analysis of $\Delta \Delta \mathrm{G}$ scores involving high and low occupancy microRNAs revealed a striking difference between the two groups; the distribution of $\Delta \Delta \mathrm{G}$ scores across the microRNA $\bullet$ mRNA pairs involving the high occupancy microRNAs is significantly skewed toward more negative values compared with pairs involving the low occupancy microRNAs (Fig. 8A, top vs. bottom panels,
A

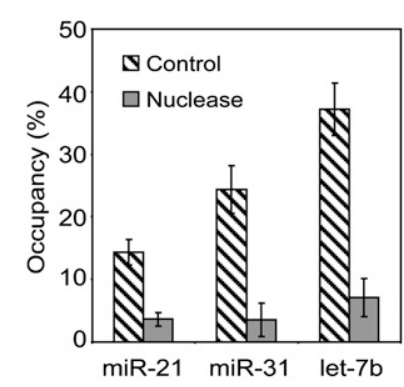

C

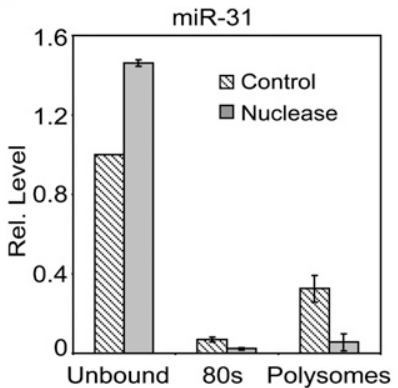

B

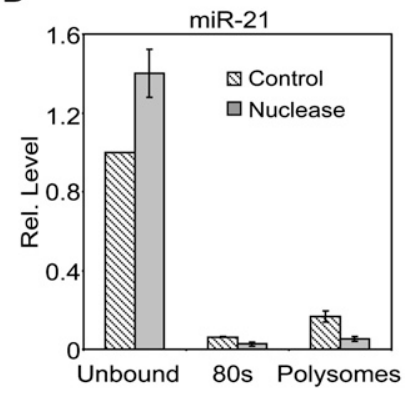

$\mathrm{D}$

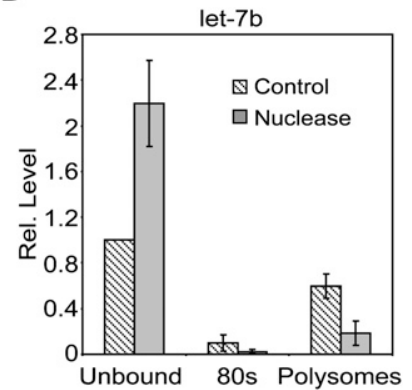

FIGURE 7. High occupancy of microRNAs reflects their binding to the mRNA targets and not to the $80 \mathrm{~S}$ ribosomal subunits. $(A)$ Polysome occupancy of miR-21, miR-31, and let-7b with and without micrococcal nuclease treatment of hESCs (nuclease and control, respectively). Note the decrease in the occupancy of all tested microRNAs following nuclease treatment. $(B-D)$ Relative abundance of miR-21 $(B)$, miR-31 $(C)$, and let-7b $(D)$ in unbound, 80S, and polysome fractions with and without micrococcal nuclease treatment. Levels are relative to the abundance in the unbound fraction of untreated hESC (control). Note the negligible levels in the $80 \mathrm{~S}$ fraction following nuclease treatment. Values displayed as mean $+/-\mathrm{SE}$. respectively). Statistical analysis of the likelihood of observing such differences in randomly selected groups of four microRNAs revealed that the observed distinction between the high and low occupancy microRNAs is highly significant $\left(P\right.$-value $<1.5 \times 10^{-6}$ based on permutation test for the average difference in $\Delta \Delta \mathrm{G}$ ) (Fig. 8B). Additional analysis of microRNA occupancy as a function of $\Delta \Delta \mathrm{G}$ revealed a clear anti-correlation (more negative $\Delta \Delta \mathrm{G}$ leads to higher occupancy, correlation $=-0.64$ ) (Fig. 8B, inset). Separate analysis for $\Delta G_{-}$duplex (Supplemental Fig. S6A) and $\Delta$ G_open (Supplemental Fig. S6B) showed that the difference in $\Delta \Delta \mathrm{G}$ between high and low occupancy microRNAs is mostly due to the duplex energy; the average

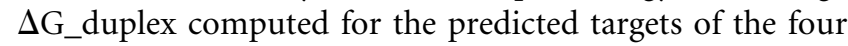
high occupancy microRNAs was significantly more negative than the $\Delta G$ _duplex for predicted targets of the four low occupancy microRNAs (Supplemental Fig. S6A). In contrast, the $\Delta G_{-}$open averages for the two groups of microRNAs were much less distinguishable (Supplemental Fig. S6B).

To determine if this difference holds for experimentally identified targets of the microRNAs, we overexpressed the mature forms of let-7b and miR-598 (high and low occupancy, respectively) and analyzed genome-wide changes in the mRNA levels compared to overexpression of a negative control. Of the predicted targets of let-7b and miR-598, we identified genes that were also down-regulated by the respective overexpression, and we considered these genes as "empirical targets." Here again, the distribution of $\Delta \Delta \mathrm{G}$ scores of let-7b was considerably and significantly skewed toward negative values $\left(P\right.$-value $\left.<1 \times 10^{-7}\right)$ (Fig. 8C), indicating more favorable interactions between the let- $7 \mathrm{~b}$ and its empirically identified targets compared to the interactions between miR-598 and its targets.

To seek functional support for the dependence of microRNA occupancy on the interaction energy, we analyzed changes in $\Delta \Delta G$ following seed swapping in let- $7 \mathrm{~b}$ and miR-598. Replacement of let-7b seed with the seed of miR-598 shifted the $\Delta \Delta \mathrm{G}$ toward less negative values (Fig. $8 \mathrm{D}$, light blue) and reduced the occupancy of the let- $7 \mathrm{~b}$ (Fig. 6B). As expected, the inverse replacement of miR-598 seed with the seed of let- $7 \mathrm{~b}$ shifted the $\Delta \Delta \mathrm{G}$ toward more negative values (Fig. 8D, pink) and increased the occupancy of miR-598 (Fig. 6B). Notably, the difference in occupancy between the endogenous let-7b and the chimeric miR-598 with let-7b seed (Fig. 6B) appeared to be larger than could be explained by the respective difference in the average $\Delta \Delta G$ computed based on the predicted targets (Fig. 8D). We, therefore, computed the respective $\Delta \Delta G$ for experimentally determined targets, identified by overexpressing the chimeric miR-598 (chimera \#2) and analyzing the genome-wide effect on mRNA levels using expression microarray. As in the case of the endogenous let- $7 \mathrm{~b}$, we identified predicted targets that were repressed by the overexpression (vs. control) and regarded them as "empir- 
A
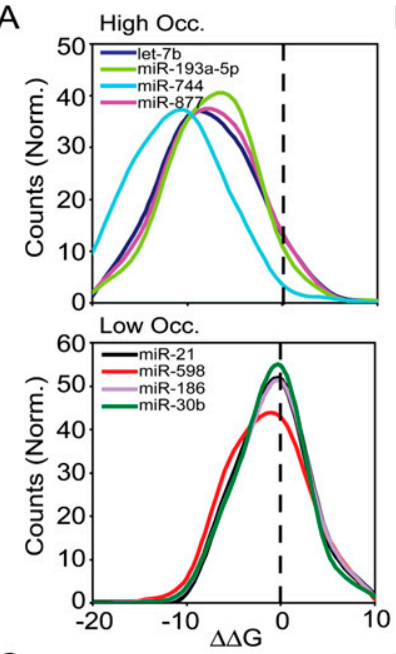

C

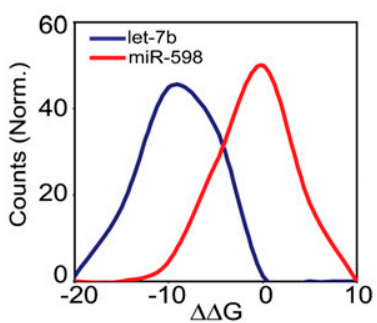

E

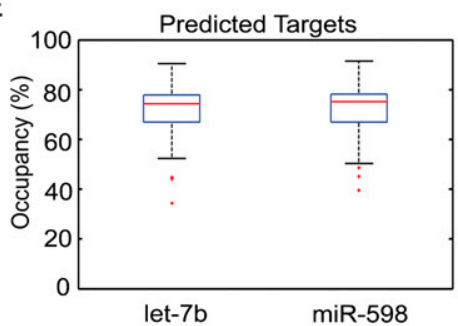

B

D
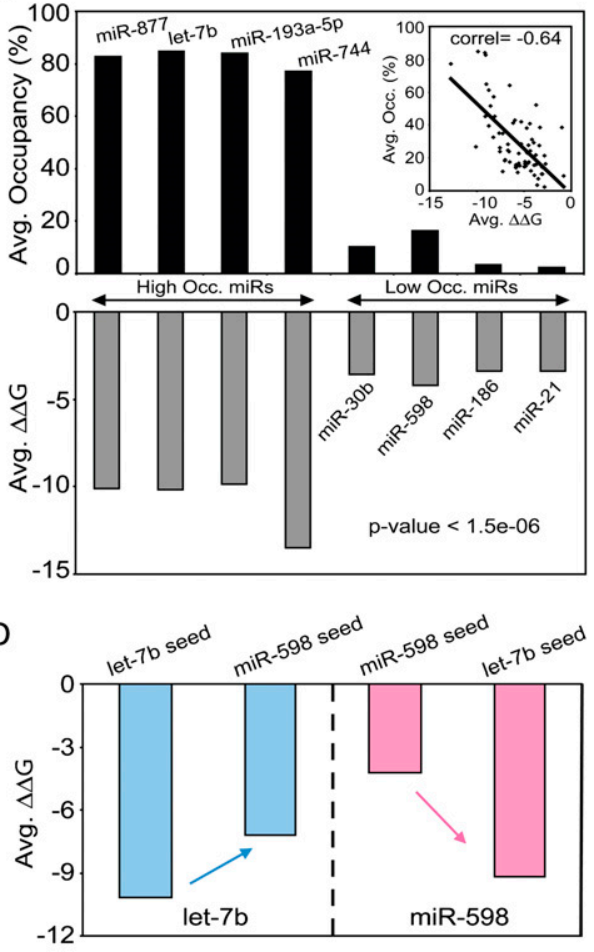

$\mathrm{F}$

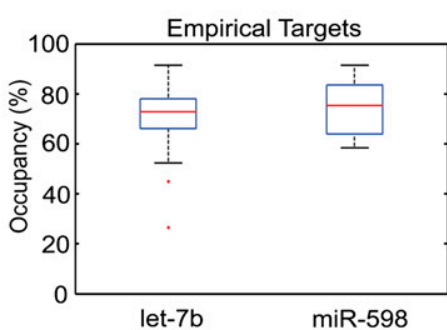

FIGURE 8. Polysome occupancy of microRNAs depends on the strength of interactions with their targets. $(A)$ Normalized histograms of $\Delta \Delta G$ scores for four very high and very low occupancy microRNAs $(>70 \%$ and $<13 \%$, respectively, in both hESCs and hFFs). Scores were computed by the PITA algorithm for each microRNA and its predicted mRNA targets. (B) Polysome occupancy (averaged across hESCs and hFFs) and average $\Delta \Delta \mathrm{G}$ of the microRNAs in $A$. Inset displays the average occupancy vs. average $\Delta \Delta G$ for all the microRNAs that were detected in both hESCs and hFFs. Significance of the difference in average $\Delta \Delta \mathrm{G}$ between the high and low occupancy microRNAs was computed based on permutation test, $P$-value $<1.5 \times 10^{-6}$. (C) Same as $A$ for empirical mRNA targets of let-7b and miR-598, representing high and low occupancy microRNA, respectively. (D) Functional analysis of the effect of seed swapping on $\Delta \Delta \mathrm{G}$. Replacement of let-7b seed with the seed of miR-598 (light blue) shifted the average $\Delta \Delta \mathrm{G}$ of let-7b toward less negative values (blue arrow) and reduced the occupancy (Fig. 6B). Conversely, replacement of miR-598 seed with the seed of let-7b shifted the average $\Delta \Delta \mathrm{G}$ toward more negative values (pink arrow) and increased the occupancy (Fig. 6B). (E) Box plot distributions of occupancy values of the top $100 \mathrm{mRNA}$ targets predicted by the PITA algorithm for let-7b and miR-598. $(F)$ Box plot distributions of occupancy values of empirical mRNA targets of let-7b and miR-598, respectively. Displayed occupancies in $E$ and $F$ were measured in hESCs.

ical targets" (listed in Supplemental file, "Empirical targets for ddG.xls"). We then analyzed the average $\Delta \Delta G$ for the chimeric miR-598 and its empirical targets and compared it to the endogenous let- $7 \mathrm{~b}$ and its empirical targets. We found that the $\Delta \Delta \mathrm{G}$ for the endogenous let- $7 \mathrm{~b}$ and its empirical targets is significantly more negative than that of the chimeric miR598 and its empirical targets $(P$-value $<$ $1 \times 10^{-4}$ ) (Supplemental Fig. S7). This difference in $\Delta \Delta \mathrm{G}$ is in line with, and could potentially explain, the observed difference in occupancy between the endogenous let- $7 \mathrm{~b}$ and the chimeric miR-598 with let-7 seed (Fig. 6B).

Since the overall interaction of microRNAs with their targets is also affected by the number of targets and the number of microRNA sites on the targets, we computed these numbers for predicted targets and sites of the selected four high and low occupancy microRNA. However, unlike the clear distinction in the $\Delta \Delta \mathrm{G}$ between the high and low occupancy microRNAs, the number of predicted targets and sites was highly variable within each group and could not account for the difference between the groups $(P$-value $=0.22$ and 0.24 for targets and sites, respectively) (Supplemental Table S3).

\section{Preferences for high and low polysome occupancy of microRNAs do not reflect preferences of their targets}

To determine if the occupancy of mRNA targets might also contribute to the occupancy of the microRNAs, we profiled the occupancy of predicted and empirical targets of miR-598 and let-7b, representing, respectively, low and high occupancy microRNAs. We fractionated the RNA into polysome-bound and unbound fractions, spiked in exogenous control mRNAs (Affymetrix Eukaryotic Poly-A control mix), and measured genome-wide mRNA levels in each fraction by hybridization to Affymetrix arrays (Human Gene 1.0 ST). Analysis of polysome occupancies of the empirical targets of let-7b and miR-598 in hESCs revealed no difference (Fig. 8F). Similar results were obtained for the predicted targets (Fig. 8E) and for predicted targets of other microRNAs (data not shown). Additionally, the difference in occupancy could not be attributed to the average expression of the predicted and empirical targets (Supplemental Table S4).

Overall, these results show that the preference for low and high polysome occupancy of microRNAs depends on 
the affinity of the microRNAs for their targets and does not reflect differential occupancy of these targets.

\section{DISCUSSION}

While some microRNAs have been shown to cosediment with polysomes (Olsen and Ambros 1999; Nelson et al. 2004; Kim et al. 2004; Maroney et al. 2006; Nottrott et al. 2006; Jannot et al. 2011), the degree of microRNA association with polysomes has not yet been quantified in any cell type. It, therefore, remained unclear whether microRNAs have distinct tendencies for associating with polysomes and whether these tendencies are influenced by properties of the microRNA or the cellular context. Here, we used a highthroughput method to measure polysome occupancies of microRNAs in a single multiplexed assay. It enables quantification of polysome occupancies of all microRNAs at once, thus allowing direct comparison between microRNAs in a given cellular context. Our measurements revealed a wide range of microRNA degrees of association with polysomes, with most microRNAs exhibiting low to medium association (occupancies of $0 \%-40 \%$ ) and fewer exhibiting considerably higher association (occupancy $>60 \%$ ). The observed preferences of individual microRNAs for low, medium, and high association with polysomes were similar in two very different cell types (hESCs and hFFs) and were independent of the abundance of the microRNAs. Previous work in HeLa cells reported copurification with polysomes of three randomly chosen microRNAs (let-7, mir-21, and miR-16) and hypothesized that association with polysomes is general for most microRNAs (Maroney et al. 2006). We found that two of these microRNAs, miR-16 and miR-21, exhibit considerably lower polysome occupancy compared with let-7b in both hESCs and hFFs. These quantitative measurements indicate that the degree of microRNA association with polysomes is microRNA-specific.

Polysome occupancy of microRNAs may also depend on the state or type of the cells. Indeed, some studies (Maroney et al. 2006; Nottrott et al. 2006) showed that the sedimentation of microRNAs with polysomes decreases upon treatments which block translation initiation or elongation. We verified this in hFFs by measuring the occupancy of a few microRNAs following treatment with rapamycin. Unlike the effect of global translation inhibition, which is expected to decrease the polysome occupancy of all microRNAs, changes in cell type may affect only a specific subset of microRNAs. This possibility was supported by the few occupancy differences that we detected in hESCs and hFFs for some of the microRNAs (Fig. 2B) and might reflect changes in the relative abundance of microRNA targets (see below). While we cannot exclude the possibility of broader differences in other cell types, our measurements show that most microRNAs retained their preference for high and low association with polysomes in two very different cell types, hESCs and hFFs.
Since microRNAs interact with the RNA-induced silencing complex (RISC), their recruitment to polysomes may be affected by factors which regulate the association of the RISC with polysomes. For example, recent work has identified RACK-1 as an evolutionarily conserved molecular adaptor which recruits regulators of mRNA translation into the ribosomes (Jannot et al. 2011). RACK1 binds KSRP, a member of the Dicer complex, and is required for the recruitment of mature microRNAs to the RISC (Otsuka et al. 2011). RACK1 was also shown to interact with Ago-2 protein (Jannot et al. 2011; Otsuka et al. 2011). Alteration of RACK1 expression alters microRNA function and impairs the association of the RISC with translating ribosomes (Jannot et al. 2011). Furthermore, RACK1 RNAi in Caenorhabditis elegans has been shown to affect the recruitment of let-7 into polysomes but does not affect the polysome association of let-7 targets, such as lin- 41 and daf-12 (Jannot et al. 2011). Still, adaptors like RACK1 that modulate the association of the RISC with polysomes may influence the recruitment of all microRNAs into polysomes and are not necessarily responsible for the differential occupancy of microRNAs.

What is, therefore, the mechanistic basis of microRNAspecific tendencies to associate with polysomes? We showed, using functional assays (overexpression of endogenous and chimeric microRNAs) and bioinformatics analysis, that the tendency for high and low polysome occupancy of a microRNA could be specified by its mature form and is strongly influenced by the choice of seed. This supports a role of the mRNA targets of the microRNA in specifying its association with polysomes. Consistent with this hypothesis, we showed that disruption of polysomes by nuclease treatment of the target mRNA shifts the occupancy of microRNAs toward the unbound fraction as opposed to the monosome, $80 \mathrm{~S}$ fraction (Fig. 7B-D). This shows that high occupancy of microRNAs does not reflect microRNA-specific interactions with the ribosomal RNA (in line with previous studies which demonstrated association of microRNAs with polysomes undergoing active translation [Olsen and Ambros 1999; Maroney et al. 2006; Nottrott et al. 2006]). Interaction of microRNAs with their targets may account for their association with polysomes but does not necessarily lead to microRNA-specific differences in occupancy. The latter might, however, be explained by microRNA-specific differences in the strength of pairing interactions with the respective targets. Indeed, we showed that the interactions between very high occupancy microRNAs and their empirically identified (or predicted) targets are considerably stronger compared to the interactions involving low occupancy microRNAs (Fig. 8). Differences in occupancy and $\Delta \Delta \mathrm{G}$ in chimeric microRNAs further provided functional support for the dependence of occupancy on the strength of pairing interactions (Fig. 8D). Since most of the mRNAs reside primarily in polysomes (Fig. 8E,F; Arava et al. 2003; Hendrickson et al. 2009), stronger interaction between the 
microRNA and its targets promotes higher association of the microRNA with polysomes. Hence, microRNA-specific affinities to the mRNA targets can provide a mechanistic basis for microRNA-specific occupancies. This model can also account for cell type-dependent differences in the occupancy of some of the microRNAs. Indeed, different cells may express different sets of targets of a given microRNA and thereby influence the recruitment of this microRNA into polysomes. Specifically, we expect the recruitment of a given microRNA into polysomes to depend on a weighted sum of interaction strengths, where the weights correspond to the expression of each target. For most microRNAs, the influence of mRNA expression appears to be relatively small, probably because each microRNA could potentially target many mRNAs, so the change in expression of individual targets tends to be averaged out. Nonetheless, if the number of expressed targets of a given microRNA is small, the influence of expression changes on recruitment of the microRNA to polysomes might be larger compared to the case of many targets. The occupancy of the microRNA in these cases might, therefore, exhibit increased sensitivity to the expression levels of the targets. This may explain the difference in occupancy we observed in hESCs versus hFFs for some of the microRNAs (Fig. 2B). Similar considerations apply to the potential scenario in which the targets are depleted from cells that express the matching microRNAs (Farh et al. 2005; Stark et al. 2005; Mishima et al. 2009). This may lead to reduced recruitment of the microRNAs into polysomes and to increased sensitivity to changes in mRNA expression. We, therefore, conclude that differential occupancy of microRNAs depends on microRNAspecific affinities to the targets and can be modulated by the relative expression of the targets. This explains why the mature form of the microRNA suffices to specify its tendency for high or low polysome occupancy and why this occupancy is independent of the abundance of the microRNA. It also accounts for the observed influence of the seed on the occupancy of the microRNA, as much of the interaction is in the seed region.

What could be the functional significance of low and high polysome occupancy of microRNAs? Association of microRNAs with polysomes has been hypothesized to reflect involvement in translation repression (Olsen and Ambros 1999; Kim et al. 2004; Nelson et al. 2004; Nottrott et al. 2006). This was supported, in part, by demonstrating that let-7 cosediments with polysomes and represses protein production at the level of translational initiation (Pillai et al. 2005) and elongation (Nottrott et al. 2006). These studies showed that let-7 shifted the sedimentation of its mRNA targets to lighter polysomes. Additional studies implicated let-7 and other microRNAs in widespread repression by degradation of the mRNAs of their targets (Lim et al. 2005; Guo et al. 2010). How the relative extent of mRNA degradation and translation inhibition is determined is largely unknown. Since it is possible that the extent of involvement in translation repression depends on the degree of microRNA association with polysomes, our study may provide a new perspective for investigating microRNA's mechanisms of repression.

\section{MATERIALS AND METHODS}

\section{Cell culture and transfections}

The H9 line of human embryonic stem cells was obtained from WiCell (Wisconsin International Stem Cell Bank, WiCell Research Institute). hESCs were maintained in the Stem Cell Core Unit, Weizmann Institute of Science in Israel and propagated (passages 35-55) on irradiated mouse embryonic fibroblasts in hESC medium composed of DMEM:F12(HAM) (Biological Industries), $20 \%$ knockout serum replacement, $1 \%$ penicillin-streptomycinglutamine, 1\% MEM-nonessential amino acids, 0.2\% 2-mercaptoethanol (all from Gibco, Invitrogen) and $8 \mathrm{ng} / \mathrm{mL}$ bFGF (Peprotech, Inc., cat. \#100-18B). Cells were passaged every 3-4 d using $0.1 \%$ collagenase (Gibco, Invitrogen, cat. \#17104-019). For selfrenewing feeder-free conditions, cells were transferred to dishes precoated with growth factor (GF)-reduced matrigel (BD Biosciences, cat. \#356231) and treated with hESC medium (including bFGF) supplemented with conditioned medium. The latter was collected from 24-h cultures of irradiated mouse embryonic fibroblasts (MEFs). Experiments with hESCs were performed $72 \mathrm{~h}$ after plating. For analysis of differentiated hESCs (Supplemental Fig. S3), hESCs were plated in matrigel-coated dishes and were treated for $10 \mathrm{~d}$ with $0.5 \mu \mathrm{M}$ of all-trans retinoic acid (Sigma Aldrich) in hESC medium lacking bFGF.

Transfections of hESCs were performed using Dharmafect reagent \#2 (Dharmacon, Thermo Scientific, cat. \#T-2002-03) after $48 \mathrm{~h}$ in feeder-free conditions. Cells were transfected for $24 \mathrm{~h}$ with $1 \mu \mathrm{M}$ of the following constructs (from Ambion): hsa-let-7b (UGAGGUAGUAGGUUGUGUGGUU, PM11050), hsa-miR-598 (UACGUCAUCGUUGUCAUCGUCA, PM11417), and pre-negative control\#1 (AM17110). These constructs are small, double-stranded RNAs that mimic mature endogenous microRNAs.

Human foreskin fibroblasts were kindly provided by Prof. Joseph Itskovitz-Eldor. hFFs were grown in medium composed of DMEM, 10\% fetal bovine serum (both from Biological Industries), and $1 \%$ penicillin-streptomycin-glutamine (Gibco). Cells were passaged every 6-8 d using Trypsin solution B (Biological Industries) until passage 20 .

For the translation inhibition experiment, hFFs were treated for $4 \mathrm{~h}$ with $100 \mathrm{nM}$ of rapamycin (Toronto Research Chemicals Inc. cat. \#R124000) diluted in DMSO (Sigma), or with DMSO alone as a control.

\section{Polysome profiling analysis and RNA purification}

\section{Lysate extraction}

Cells were treated with $0.1 \mathrm{mg} / \mathrm{mL}$ cycloheximide (Calbiochem, cat. \#239764), placed immediately on ice, washed twice with icecold "Pre-buffer" (20 mM Tris-HCl, $\mathrm{pH}$ 8.0, $5 \mathrm{mM} \mathrm{MgCl}_{2}, 140$ $\mathrm{mM} \mathrm{KCl}$, and $0.1 \mathrm{mg} / \mathrm{mL}$ cycoheximide), and resuspended in lysis buffer $(0.1 \%$ Brij [Fluka, cat. \#16004], 0.1\% deoxycholate, 0.22 $\mathrm{mg} / \mathrm{mL}$ heparin, $0.5 \mathrm{mM}$ DTT, $0.1 \mathrm{U} / \mu \mathrm{L}$ SuperAsin [Ambion 
cat. \#AM2696] and $5 \mu \mathrm{g} / \mathrm{mL}$ protease inhibitor mix). The lysate was homogenized by vortex at high speed for several times over 20 min and centrifuged first for $10 \mathrm{~min}$ at $3500 \mathrm{rpm}$ and then for another $10 \mathrm{~min}$ at $9500 \mathrm{rpm}$. The supernatant was snap-frozen for later use.

\section{Micrococcal nuclease treatment}

Cytoplasmic extracts of hESCs were treated with micrococcal nuclease $\left(300 \mathrm{U} / \mu \mathrm{L}\right.$, Fermentas) in the presence of $2 \mathrm{mM} \mathrm{CaCl}_{2}$ for $30 \mathrm{~min}$ at $20^{\circ} \mathrm{C}$. The reaction was stopped by the addition of EDTA to a final concentration of $2.5 \mathrm{mM}$, and the extracts were loaded on the sucrose gradients.

\section{Sucrose gradient velocity sedimentation}

Ten percent and $60 \%$ sucrose solutions were dissolved in "Prebuffer" with the addition of $0.5 \mathrm{mM}$ DTT, and 10\%-60\% linear sucrose gradients were prepared using Biocomp Gradient Master according to the manufacturer's recommendations. Approximately $150 \mu \mathrm{g}$ of lysate was layered onto the gradient and centrifuged in a SW-41 rotor at 41,000 rpm for $1.5 \mathrm{~h}$. Absorbance of the gradient at $260 \mathrm{~nm}$ was monitored using a UV6 system (Teledyne-Isco), and the following fractions were collected: (1) unbound pool starting from top of the gradient until 80S; and (2) polysomal pool starting immediately after $80 \mathrm{~S}$ until the end of the gradient. To confirm that the exclusion did not affect the measured parameters, the $80 \mathrm{~S}$ fraction was collected separately and subjected to further analysis, similar to the other fractions. For higher resolution analysis of the polysome profile, the polysomal pool was collected in separate fractions ( $\sim 1 \mathrm{~mL}$ each, total of seven fractions) and subjected to further analysis of the abundance of individual microRNAs.

\section{RNA extraction}

miR-488* was spiked at $1.3 \mathrm{ng} / \mu \mathrm{L}$ into the bound and unbound fractions and then used as an exogenous control microRNA for normalizing the levels of each fraction prior to estimation of occupancy. For measuring polysome occupancy of mRNAs, we used the Affymetrix GeneChip Eukaryotic Poly-A control mix as exogenous, spike-in control mRNAs (diluted 1:15,000). Total RNA was isolated from each pool using the PureLink RNA mini kit (Invitrogen, cat. \#12183018A), with an additional volume of pure isopropanol (final volume of $70 \%$ ) required for efficient extraction of small RNAs. For measuring microRNA expression levels, we isolated total RNA using the RNeasy Plus mini kit (Qiagen, cat. \#74104).

\section{Reverse transcription and real-time PCR}

Measuring the total abundance of microRNAs and their levels in the unbound and polysomal fractions was performed using highthroughput real-time qPCR. Purified RNA was reverse-transcribed using the TaqMan microRNA Reverse Transcription kit and Megaplex RT primers (Applied Biosystems). The diluted RT product was loaded on TaqMan Array Human MicroRNA Cards v2.0 pool A and B (Applied Biosystems) and analyzed on a 7900HT Fast Real-Time PCR system (Applied Biosystems). The optimal threshold was set for each detector, and the respective $C_{T}$ values were recorded. $C_{T}$ values of the exogenous or endogenous controls (miR-488* for occupancy measurements and RNU48 for total abundance, respectively) were subtracted from the $\mathrm{C}_{\mathrm{T}}$ values of each probe (probes for both the endogenous and exogenous controls were included on the TaqMan Array Cards). Subtracted values were used to compute the polysome occupancy and total abundance of each microRNA. Measurements for individual microRNAs (hsa-let-7b, hsa-miR-598, hsa-miR-21, hsa-miR-31) were performed with specific TaqMan RT and Real-Time primers (Applied Biosystems), using the same exogenous and endogenous controls (hsa-miR-488* and hsa-RNU48, respectively). All analyses involving occupancy were restricted to microRNAs that were detected at cycles below $35\left(\mathrm{C}_{\mathrm{T}}<35\right)$ in both ribosome-free and polysome-bound.

\section{Analysis of microRNA polysome occupancy}

Occupancy measurements were based on comparison of the normalized levels of each microRNA in the translated (polysomal) fraction and the untranslated (unbound) fraction. We defined the occupancy of each microRNA as the ratio between its normalized level in the polysomal fraction and the sum of its levels in both fractions (Fig. 1A).

\section{Microarray analysis}

mRNA from the unbound and polysomal fractions was analyzed (after adding the spiked-in control) by Affymetrix Human Gene 1.0 ST microarrays. Each sample was processed according to the manufacturer's instructions using Affymetrix GeneChip Whole Transcript Sense Target Labeling Assay. Hybridization and scanning of the microarrays were performed at the microarray core facility in the Weizmann Institute of Science, Israel. Following scanning, the arrays were transformed to a processed image, which was converted to one fluorescence intensity value per probe, using Affymetrix GeneChip Operating Software (GCOS). Data was analyzed using Partek Genomics Suite 6.5 (Partek Inc.). Initial normalization of probe intensity across arrays was performed using the RMA (robust multichip analysis) algorithm. The value of each gene was then further normalized by the average probe intensity values of the spiked-in controls. Polysome occupancy of each mRNA was computed from the normalized values similarly to the microRNA case. The data set is available through GEO, Series entry GSE31768.

\section{Chimeric microRNA design and measurement}

Chimeric microRNA constructs (chemically modified doublestranded RNAs that mimic mature microRNAs) were generated as follows: For chimera \#1, the seed of mature hsa-let-7b (nucleotides 2-8 from the $5^{\prime}$ end) was swapped with the seed of hsa-miR598 (chimera \#1, UACGUCAUUAGGUUGUGUGGUU) and vice versa for chimera \#2 (UGAGGUAGCGUUGUCAUCGUCA). Both constructs were custom-made by Applied Biosystems and transfected into hESCs as described for the transfection of endogenous microRNAs. The levels of the chimeric microRNAs were measured using respective, custom-made Taqman probes (Applied Biosystems).

\section{Analysis of free energy of microRNA.mRNA pairs}

Differences in free-energy scores $\left(\Delta \Delta \mathrm{G}, \Delta \mathrm{G} \_\right.$duplex, and $\Delta \mathrm{G} \_$open $)$ were computed using the PITA algorithm for each microRNA•mRNA 
pair (Kertesz et al. 2007). For each microRNA, we identified a set of predicted targets using the following criteria: 7- or 8-mer site, no G:U wobble in the 7- or 8-mer, up to one mismatch in the seed, and without requiring conservation. For each mRNA target (listed in the Supplemental file, "Targets for ddG.xls"), we identified the site with the lowest $\Delta \Delta \mathrm{G}$ score and selected this site to represent the target. For each microRNA and its set of targets (including the chimeric microRNs), we computed the $\Delta \Delta \mathrm{G}$, $\Delta \mathrm{G} \_$duplex, and $\Delta \mathrm{G} \_$open scores and analyzed differences between microRNAs. To determine if the differences in $\Delta \Delta \mathrm{G}$ correlate with the differences in occupancy, we selected two groups of four microRNAs that exhibited very high $(>70 \%)$ or very low occupancy $(<13 \%)$ in both hESCs and hFFs. For testing the statistical significance of this correlation (Fig. 8B), we computed the average $\Delta \Delta \mathrm{G}$ for each of two groups of four microRNAs that were randomly selected and repeated this calculation $10^{8}$ times. We then counted the number of times, $n$, in which the average $\Delta \Delta \mathrm{G}$ difference between the groups of four randomly selected microRNAs exceeded the difference observed by the specific groups of high and low occupancy microRNAs. The resulting $P$-value is given by $n / 10^{8}$. Significance of the difference between distributions of $\Delta \Delta G$ corresponding to specific microRNAs (e.g., in Fig. 8C) was determined by the Kolmogov-Smirnov test.

\section{SUPPLEMENTAL MATERIAL}

Supplemental material is available for this article.

\section{ACKNOWLEDGMENTS}

We gratefully thank Amit Zeisel (Weizmann Institute of Science, WIS), Tomer Handelman (Tel-Aviv University, TAU), and Ohad Manor (WIS) for assistance in bioinformatics analyses. We thank Dr. Eran Hornstein (WIS) and Dr. Arkady Torchinsky (TAU) for critical reading and helpful comments on the manuscript. We also thank the members of our laboratory, particularly Michael Elgart, Haggai Kaspi, Yogev Sela, and Shay Stern for helpful discussions and technical help. We thank Prof. Joseph ItskovitzEldor (Rambam-Technion Stem Cell Research Center, Israel) for providing the human foreskin fibroblasts (hFFs) used in this study. We thank Dr. Elena Ainbinder (Stem Cell Core Facility Unit, Weizmann Institute of Science, Israel) for support in the hESC work. This work was supported by The Israel Science Foundation (grant No. 1541/09) and by a Wolfson Family Charitable Trust (2009-10). Y.S. was supported by a CDA award from the Human Frontier Science Organization (CDA0063/2007-C). Y.S. is Incumbent of the Daniel E. Koshland Sr. Career Development Chair at the Weizmann Institute.

Received March 5, 2012; accepted May 29, 2012.

\section{REFERENCES}

Ambros V. 2004. The functions of animal microRNAs. Nature 431: 350-355.

Arava Y, Wang Y, Storey JD, Liu CL, Brown PO, Herschlag D. 2003. Genome-wide analysis of mRNA translation profiles in Saccharomyces cerevisiae. Proc Natl Acad Sci 100: 3889-3894.

Baek D, Villen J, Shin C, Camargo FD, Gygi SP, Bartel DP. 2008. The impact of microRNAs on protein output. Nature 455: 64-71.
Bagga S, Bracht J, Hunter S, Massirer K, Holtz J, Eachus R, Pasquinelli AE. 2005. Regulation by let-7 and lin-4 miRNAs results in target mRNA degradation. Cell 122: 553-563.

Bartel DP, Chen CZ. 2004. Micromanagers of gene expression: The potentially widespread influence of metazoan microRNAs. Nat Rev Genet 5: 396-400.

Brennecke J, Stark A, Russell R, Cohen S. 2005. Principles of microRNAtarget recognition. PLoS Biol 3: e85. doi: 10.1371/journal.pbio. 0030085.

Carthew RW. 2006. Gene regulation by microRNAs. Curr Opin Genet Dev 16: 203-208.

Chen C, Ridzon DA, Broomer AJ, Zhou Z, Lee DH, Nguyen JT, Barbisin M, Xu NL, Mahuvakar VR, Andersen MR, et al. 2005. Real-time quantification of microRNAs by stem-loop RT-PCR. Nucleic Acids Res 33: e179. doi: 10.1093/nar/gnil78.

Doench J, Sharp P. 2004. Specificity of microRNA target selection in translational repression. Genes Dev 18: 504-511.

Farh KK, Grimson A, Jan C, Lewis BP, Johnston WK, Lim LP, Burge CB, Bartel DP. 2005. The widespread impact of mammalian microRNAs on mRNA repression and evolution. Science 310: 1817-1821.

Filipowicz W, Bhattacharyya SN, Sonenberg N. 2008. Mechanisms of post-transcriptional regulation by microRNAs: Are the answers in sight? Nat Rev Genet 9: 102-114.

Griffiths-Jones S. 2006. miRBase: The microRNA sequence database. Methods Mol Biol 342: 129-138.

Griffiths-Jones S. 2007. Annotating noncoding RNA genes. Annu Rev Genomics Hum Genet 8: 279-298.

Griffiths-Jones S, Saini HK, van Dongen S, Enright AJ. 2008. miRBase: Tools for microRNA genomics. Nucleic Acids Res 36: D154-D158.

Grimson A, Farh KK, Johnston WK, Garrett-Engele P, Lim LP, Bartel DP. 2007. MicroRNA targeting specificity in mammals: Determinants beyond seed pairing. Mol Cell 27: 91-105.

Guo H, Ingolia NT, Weissman JS, Bartel DP. 2010. Mammalian microRNAs predominantly act to decrease target mRNA levels. Nature 466: 835-840.

Hendrickson DG, Hogan DJ, McCullough HL, Myers JW, Herschlag D, Ferrell JE, Brown PO. 2009. Concordant regulation of translation and mRNA abundance for hundreds of targets of a human microRNA. PLoS Biol 7: e1000238. doi: 10.1371/journal.pbio. 1000238.

Jackson RJ, Standart N. 2007. How do microRNAs regulate gene expression? Sci STKE 2007: re1. doi: 10.1126/stke.3672007re1.

Jannot G, Bajan S, Giguere NJ, Bouasker S, Banville IH, Piquet S, Hutvagner G, Simard MJ. 2011. The ribosomal protein RACK1 is required for microRNA function in both $C$. elegans and humans. EMBO Rep 12: 581-586.

Kertesz M, Iovino N, Unnerstall U, Gaul U, Segal E. 2007. The role of site accessibility in microRNA target recognition. Nat Genet 39: $1278-1284$.

Kim J, Krichevsky A, Grad Y, Hayes GD, Kosik KS, Church GM, Ruvkun G. 2004. Identification of many microRNAs that copurify with polyribosomes in mammalian neurons. Proc Natl Acad Sci 101: 360-365.

Lim LP, Lau NC, Garrett-Engele P, Grimson A, Schelter JM, Castle J, Bartel DP, Linsley PS, Johnson JM. 2005. Microarray analysis shows that some microRNAs downregulate large numbers of target mRNAs. Nature 433: 769-773.

Maroney PA, Yu Y, Fisher J, Nilsen TW. 2006. Evidence that microRNAs are associated with translating messenger RNAs in human cells. Nat Struct Mol Biol 13: 1102-1107.

Meister G. 2007. miRNAs get an early start on translational silencing. Cell 131: 25-28.

Melamed D, Eliyahu E, Arava Y. 2009. Exploring translation regulation by global analysis of ribosomal association. Methods 48: 301305.

Mishima Y, Abreu-Goodger C, Staton AA, Stahlhut C, Shou C, Cheng C, Gerstein M, Enright AJ, Giraldez AJ. 2009. Zebrafish miR-1 and 
miR-133 shape muscle gene expression and regulate sarcomeric actin organization. Genes Dev 23: 619-632.

Nelson PT, Hatzigeorgiou AG, Mourelatos Z. 2004. miRNP:mRNA association in polyribosomes in a human neuronal cell line. RNA 10: $387-394$.

Nielsen CB, Shomron N, Sandberg R, Hornstein E, Kitzman J, Burge CB. 2007. Determinants of targeting by endogenous and exogenous microRNAs and siRNAs. RNA 13: 1894-1910.

Nilsen TW. 2007. Mechanisms of microRNA-mediated gene regulation in animal cells. Trends Genet 23: 243-249.

Nottrott S, Simard MJ, Richter JD. 2006. Human let-7a miRNA blocks protein production on actively translating polyribosomes. Nat Struct Mol Biol 13: 1108-1114.

Olsen PH, Ambros V. 1999. The lin-4 regulatory RNA controls developmental timing in Caenorhabditis elegans by blocking LIN-14 protein synthesis after the initiation of translation. Dev Biol 216: 671-680.

Otsuka M, Takata A, Yoshikawa T, Kojima K, Kishikawa T, Shibata C, Takekawa M, Yoshida H, Omata M, Koike K. 2011. Receptor for activated protein kinase C: Requirement for efficient microRNA function and reduced expression in hepatocellular carcinoma. PLoS ONE 6: e24359. doi: 10.1371/journal.pone.0024359.
Petersen CP, Bordeleau ME, Pelletier J, Sharp PA. 2006. Short RNAs repress translation after initiation in mammalian cells. Mol Cell 21: 533-542.

Pillai RS, Bhattacharyya SN, Artus CG, Zoller T, Cougot N, Basyuk E, Bertrand E, Filipowicz W. 2005. Inhibition of translational initiation by Let-7 microRNA in human cells. Science 309: 15731576.

Raught B, Gingras A, Sonenberg N. 2001. The target of rapamycin (TOR) proteins. Proc Natl Acad Sci 98: 7037-7044.

Selbach M, Schwanhausser B, Thierfelder N, Fang Z, Khanin R, Rajewsky N. 2008. Widespread changes in protein synthesis induced by microRNAs. Nature 455: 58-63.

Stark A, Brennecke J, Bushati N, Russell RB, Cohen SM. 2005. Animal microRNAs confer robustness to gene expression and have a significant impact on 3'UTR evolution. Cell 123: 1133-1146.

Terada N, Patel H, Takase K, Kohno K, Nairn A, Gelfand E. 1994. Rapamycin selectively inhibits translation of mRNAs encoding elongation factors and ribosomal proteins. Proc Natl Acad Sci 91: 11477-11481.

Wang B, Yanez A, Novina CD. 2008. MicroRNA-repressed mRNAs contain $40 \mathrm{~S}$ but not $60 \mathrm{~S}$ components. Proc Natl Acad Sci 105: 5343-5348. 

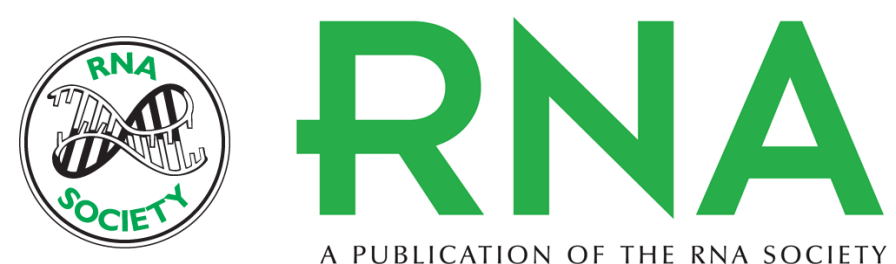

A PUBLICATION OF THE RNA SOCIETY

\section{Differential association of microRNAs with polysomes reflects distinct strengths of interactions with their mRNA targets}

Natali Molotski and Yoav Soen

RNA 2012 18: 1612-1623 originally published online July 26, 2012

Access the most recent version at doi:10.1261/rna.033142.112

\section{Supplemental http://rnajournal.cshlp.org/content/suppl/2012/06/26/rna.033142.112.DC1 \\ Material}

References This article cites 39 articles, 12 of which can be accessed free at: http://rnajournal.cshlp.org/content/18/9/1612.full.html\#ref-list-1

\section{License}

Email Alerting Receive free email alerts when new articles cite this article - sign up in the box at the Service top right corner of the article or click here.

To subscribe to RNA go to:

http://rnajournal.cshlp.org/subscriptions 